\title{
Identification for Passive Robust Fault Detection using Zonotopes*
}

\author{
Joaquim Blesa*, Vicenç Puig*, Jordi Saludes** \\ *Automatic Control Department, Technical University of Catalonia (UPC) \\ Pau Gargallo 5, 08028 Barcelona, Spain; (e-mail:\{ joaquim.blesa,vicenc.puig\}@upc.edu) \\ **Applied Mathematics Department (MA2), Technical University of Catalonia (UPC) \\ Carrer Colom 11, 08222 Terrassa, Spain (e-mail:jordi.saludes@upc.edu)
}

\begin{abstract}
In this paper, the problem of identification for passive robust fault detection when parametric modeling uncertainty is considered. In particular, a zonotope is used to bound the model parametric uncertainty. Two identification methods are introduced following, respectively, the worst-case and setmembership approaches. Then, the underlying hypothesis are discussed and performance is compared. These two identification approaches lead to two robust fault detection tests (namely, the direct and inverse tests) that are also discussed. A case study based on a four tanks system is used to exemplify the properties of the two identification and associated fault detection approaches.
\end{abstract}

Keywords: Robust fault detection, identification, interval models, zonotopes, set-membership

\section{INTRODUCTION}

The principle of model-based fault detection is to test whether the measured system inputs and outputs are consistent with the system behaviour described by a faultless model. If the measurements are inconsistent with the model of the faultless system, the existence of a fault is proved. The residual vector usually describes the consistency check between the predicted and the

\footnotetext{
* This work was supported in part by the Research Commission of the Generalitat of Catalunya (Grup SAC ref. 2005SGR00537) and by Spanish Ministry of Education (CICYT projects ref. DPI-2005-05415, ref. DPI-2006-11944 and ref. DPI2008-01996). The material of this paper was partially presented at 7th IFAC Symposium on Fault Detection, Supervision and Safety of Technical Processes, July 2009, Barcelona, Spain.
} 
real behaviour. Ideally, the residuals should only be affected by the faults. However, the presence of disturbances, noise and modeling errors causes the residuals to become nonzero and thus interferes with the detection of faults. Therefore, the fault detection procedure must be robust against these undesired effects (Chen and Patton, 1999). In case that parametric uncertainties are taken into account, the healthy system model should include a vector of uncertain parameters bounded by sets that contains all possible parameter values when the system operates normally. In the robust fault detection literature, so far, parameters have been bounded using intervals and the resulting model is known as an interval model (Puig, 2008). In case of modeling a dynamic system using an interval model, the predicted output is also bounded by an interval. Then, fault detection test is based on checking if zero is contained or not in the residual interval after propagating the parameter uncertainty to the residual (Fagarasan et al., 2004; Puig et al., 2008; Sainz et al., 2002, Ploix, 2006). Alternatively, in this paper, parameter uncertainty will be bound using a more complex shape: a zonotope, that will allow to obtain less conservative fault detection models and results. The use of zonotopes in fault detection has already been suggested in the literaure. See for example (Combastel et al, 2008; 2009).

One of the key points in model based fault detection is how models and their uncertainty bounds are obtained. Classical system identification methods provide only an estimation of the nominal model but do not provide a reliable means for bounding the uncertainty associated with the model.

This problem has been mainly stated in many papers coming from robust control field. Recently some methodologies that provides a model with its uncertainty has been developed but thinking always in its application to control (Reinelt, 2002). In fact in this community, robust system identification is used to describe the new methodologies of system identification that provide not only a nominal model but also a reliable estimate of the uncertainty associated with the model. See for example the set-membership parameter estimation algorithms proposed by Milanese (1996), that produces a set of parameters that are consistent with the model structure that has been selected and assumed noise bounds. Alternatively, Campi et al. (2009) has suggested an adaptation of classical system identifications methods in order to provide the nominal model plus the uncertainty bounds for parameters that guarantee that all collected data from the system in non-faulty scenarios will be included in the model prediction interval (worst-case parameter estimation).

The main contribution of this paper is to provide worst-case/set-membership parameter estimation algorithms using zonotopes to bound the parameter uncertainty and focusing on their application to passive robust fault detection. 
The structure of the paper is the following: Section 2 is dedicated to describe the problem, first focused on LTI systems. In Section 3, two identification approaches for robust fault detection are presented, recalling the underlying hypothesis and existing implementation algorithms. In Section 4, the fault detection tests associated to the models delivered by the proposed identification approaches are introduced. In Section 5, the performance of the two fault detection approaches in case of additive and multiplicative faults are studied. In Section 6, the extension of the indentification algorithms for LTI models to LPV models is introduced. In Section 7, a case study based on a well known control benchmark (the four-tanks system) is used to exemplify and compare the identification methods for robust fault detection presented in this paper. Finally, in Section 8 , the conclusions of the paper are provided.

\section{MODEL PARAMETRISATION}

Let us assume that the system can be expressed by an LTI model in regressor form (MA model) with additive noise as follows:

$$
y(k)=\boldsymbol{\varphi}(k) \boldsymbol{\theta}(k)+e(k)=\hat{y}(k)+e(k)
$$

where:

- $\varphi(k)$ is the regressor vector of dimension $1 \times n_{\theta}$ which can contain any function of inputs $u(k)$ and outputs ${ }^{\dagger} y(k)$.

- $\boldsymbol{\theta}(k) \in \boldsymbol{\Theta}$ is the parameter vector of dimension $n_{\theta} \times 1$

- $\boldsymbol{\Theta}$ is the set that bounds parameter values.

- $e(k)$ is the sensor additive noise bounded by a constant $|e(k)| \leq \sigma$.

In this paper, the uncertain parameter set $\boldsymbol{\Theta}$ is described by a zonotope centered in a nominal model:

$$
\boldsymbol{\Theta}=\boldsymbol{\theta}^{0} \oplus \mathbf{H B} \mathbf{B}^{n}=\left\{\boldsymbol{\theta}^{0}+\mathbf{H z}: \mathbf{z} \in \mathbf{B}^{n}\right\}
$$

where:

- $\boldsymbol{\theta}^{0} \in \mathbb{R}^{n_{\theta}}$ is the nominal model.

- $\mathbf{H} \in \mathbb{R}^{n_{\theta} \times n}$

$-\mathbf{B}^{n} \in \mathbb{R}^{n \times 1}$ is a unitary box composed by $n$ unitary $(\mathbf{B}=[-1,1])$ interval vectors.

- $\oplus$ denotes the Minkowski sum.

Notice that a particular case corresponds to the case the parameter set $\boldsymbol{\Theta}$ is bounded by an interval box:

$\boldsymbol{\Theta}=\left[\underline{\theta}_{1}, \bar{\theta}_{1}\right] \times \cdots \times\left[\underline{\theta}_{i}, \bar{\theta}_{i}\right] \times \cdots \times\left[\underline{\theta}_{n_{\theta}}, \bar{\theta}_{n_{\theta}}\right]$ where $\underline{\theta}_{i} \triangleq \theta_{i}^{0}-\lambda_{i}$ and $\bar{\theta}_{i} \triangleq \theta_{i}^{0}+\lambda_{i}$ with $\lambda_{i} \geq 0$ and $i=1, \ldots, n_{\theta}$ 
This set can be viewed as a zonotope with $\mathbf{H}$ equal to a $n_{\theta} \times n_{\theta}$ diagonal matrix:

$$
\mathbf{H}=\operatorname{diag}\left(\lambda_{1}, \lambda_{2}, \ldots, \lambda_{n_{\theta}}\right)
$$

Depending on the consideration of variation of parameters from one instant $k-1$ to the following instant $k$ two very different approaches, based on model (1), can be defined.

1. No restriction on variation in parameters, this is

$$
|\boldsymbol{\theta}(k)-\boldsymbol{\theta}(k-1)| \leq \infty, \forall k
$$

Then the parameter can vary in one time instant to the farthest parameter in the set $\Theta$, this is the worst-case variation. In the following this approach will be called worst-case approach.

This approach was first suggested by Ploix (1999) in the context of fault detection. Further works using this approach are Adrot (2000), Calafiore (2002) and Campi (2009).

2. No variation in parameter is considered, this is

$$
|\boldsymbol{\theta}(k)-\boldsymbol{\theta}(k-1)|=0, \forall k
$$

Then, the parameter is unknown but considered constant

$$
\boldsymbol{\theta}(k)=\boldsymbol{\theta}(k-1)=\boldsymbol{\theta}, \forall k
$$

Algorithms following this approach are also known as "set-membership parameter estimation" algorithms. In Milanese (1996) there is a survey of such methods.

These different approaches will be described in the following two sections.

3. WORST-CASE APPROACH

\footnotetext{
${ }^{\dagger}$ In this paper we will focus on the single output case.
} 


\subsection{Problem definition}

Given a sequence of $M$ regressor vector values $\varphi(k)$ and measurement values $y(k)$ in a fault free scenario and rich enough from the identifiability point of view. The aim is to estimate parameters and their uncertainty of a model parameterised as in Eq. (1) that can describe all the measurements $y(k)$ considering the worst-case approach, this is no restriction in parameter variation.

In this case, the set of uncertain parameters $\boldsymbol{\Theta}$ should be obtained in such a way that all measured data in a fault free scenario will be covered by the worst-case predicted output produced by using model (1) and the uncertainty parameter set ("worst-case model'), that is $y(k) \in[\underline{\hat{y}}(k)-\sigma, \overline{\hat{y}}(k)+\sigma]$ that can be rewritten as the following equations

$$
\overline{\hat{y}}(k) \geq y(k)-\sigma \text { and } \underline{\hat{y}}(k) \leq y(k)+\sigma \quad \forall k=1, \ldots, M
$$

where:

$$
\begin{aligned}
& \overline{\hat{y}}(k)=\max (\boldsymbol{\varphi}(k) \boldsymbol{\theta}(k)) \text { with } \boldsymbol{\theta}(k) \in \boldsymbol{\Theta} \\
& \underline{\hat{y}}(k)=\min (\boldsymbol{\varphi}(k) \boldsymbol{\theta}(k)) \text { with } \boldsymbol{\theta}(k) \in \boldsymbol{\Theta}
\end{aligned}
$$

Then at every instant $k$, the regressor vector $\varphi(k)$ and the measured output $y(k)$ define two half-spaces $\overline{\boldsymbol{\Theta}}_{k}$ and $\underline{\Theta}_{k}$ in $\mathbb{R}^{n_{\theta}}$ with the worst-case conditions (6) as follows: $\overline{\boldsymbol{\Theta}}_{k}=\left\{\boldsymbol{\theta} \in \mathbb{R}^{n_{\theta}}: \boldsymbol{\varphi}(k) \boldsymbol{\theta} \geq y(k)-\sigma\right\}$ and $\underline{\boldsymbol{\Theta}}_{k}=\left\{\boldsymbol{\theta} \in \mathbb{R}^{n_{\theta}}: \boldsymbol{\varphi}(k) \boldsymbol{\theta} \leq y(k)+\sigma\right\}$.

Then, the sets $\overline{\boldsymbol{\Theta}}$ and $\underline{\boldsymbol{\Theta}}$ that fulfil respectively $\boldsymbol{\varphi}(k) \boldsymbol{\theta} \geq y(k)-\sigma$ and $\boldsymbol{\varphi}(k) \boldsymbol{\theta} \leq y(k)+\sigma \quad \forall k=1, \ldots, M$ are defined by

$$
\overline{\boldsymbol{\Theta}}=\bigcap_{k=1}^{M} \overline{\boldsymbol{\Theta}}_{k} \text { and } \underline{\boldsymbol{\Theta}}=\bigcap_{k=1}^{M} \underline{\boldsymbol{\Theta}}_{k}
$$

And finally, a set $\Theta$ that fulfils both worst-case conditions (6) $\forall k=1, \ldots, M$ satisfies

$$
\boldsymbol{\Theta} \cap \overline{\boldsymbol{\Theta}} \neq \varnothing \text { and } \boldsymbol{\Theta} \cap \underline{\boldsymbol{\Theta}} \neq \varnothing
$$

The following two figures show graphically one example with $n_{\theta}=2$. 

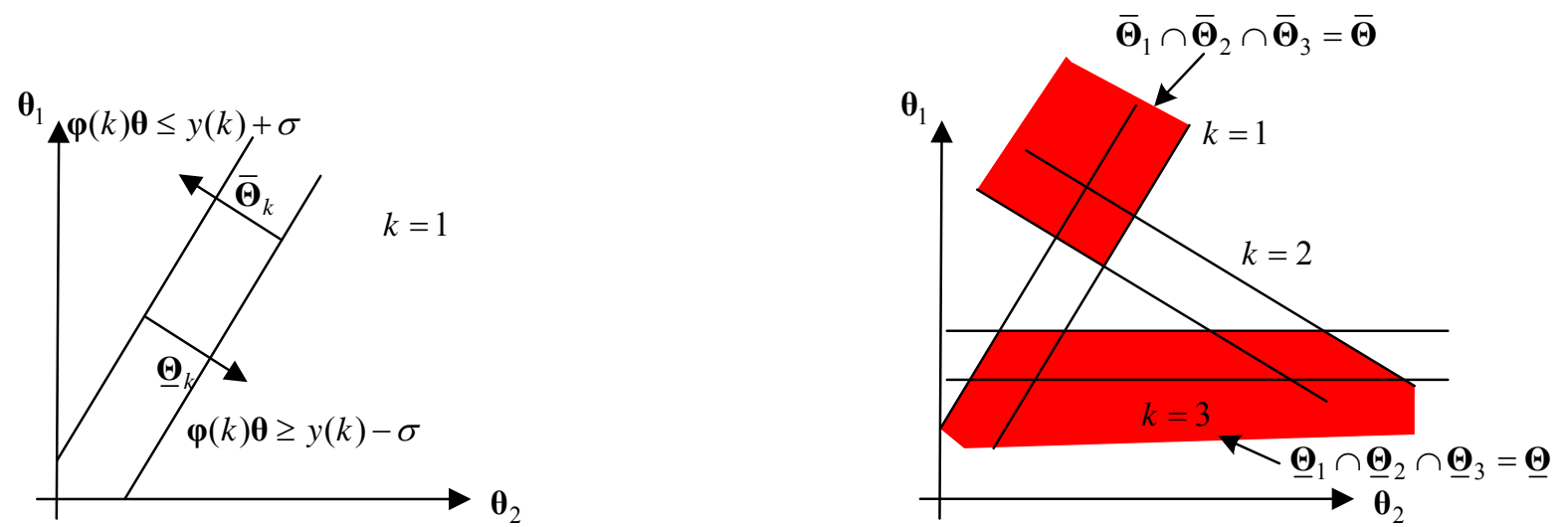

Figure 1. a) Half spaces $\overline{\boldsymbol{\Theta}}_{k}$ and $\underline{\boldsymbol{\Theta}}_{k}$ with regressor vector $\boldsymbol{\varphi}(k)$ and measured output $y(k)$ for $k=1$. b) Sets $\underline{\boldsymbol{\Theta}}$ and $\overline{\boldsymbol{\Theta}}$ with data from $k=1$ to $k=3$.

\subsection{Worst-case parameter estimation}

Considering that the parameter set $\boldsymbol{\Theta}$ can be described as the zonotope (2), the maximum (7a) and minimum prediction (7b) provided by model (1), considering worst-case variation in parameters, are given by (see Proposition 1 in Appendix)

$$
\begin{gathered}
\overline{\hat{y}}(k)=\hat{y}^{0}(k)+\|\boldsymbol{\varphi}(k) \mathbf{H}\|_{1} \\
\underline{\hat{y}}(k)=\hat{y}^{0}(k)-\|\boldsymbol{\varphi}(k) \mathbf{H}\|_{1}
\end{gathered}
$$

where $\hat{y}^{0}(k)$ is the model output prediction with nominal parameters:

$$
\hat{y}^{0}(k)=\boldsymbol{\varphi}(k) \boldsymbol{\theta}^{0} \quad \text { where } \quad \boldsymbol{\theta}^{0}=\left(\theta_{1}^{0}, \ldots, \theta_{n_{\theta}}^{0}\right)
$$

Notice that in the particular case of interval parameters follows

$$
\|\boldsymbol{\varphi}(k) \mathbf{H}\|_{1}=\sum_{i=1}^{n} \lambda_{i}\left|\varphi_{i}(k)\right|
$$

according to (3). Replacing equations (9a) and (9b) in inclusion conditions (6), the optimal zonotope that fulfills the "worstcase condition" can be computed by solving the Problem 1.

Problem 1: "Worst-case Parameter Estimation “ (general case)

$\min _{\mathbf{H}} f(\boldsymbol{\Theta}(\mathbf{H}))$
subject to: $\|\boldsymbol{\varphi}(k) \mathbf{H}\|_{1} \geq\left|y(k)-\hat{y}^{0}(k)\right|-\sigma \quad \forall k=1, \ldots, M$


In this problem, the cost function $f$ is usually the interval prediction thickness that can be calculated as

$$
\sum_{k=1}^{M}(\overline{\hat{y}}(k)-\underline{\hat{y}}(k))=2 \sum_{k=1}^{M}\|\boldsymbol{\varphi}(k) \mathbf{H}\|_{1}
$$

Problem 1 can be in general very hard to solve (Campi, 2009). In order to reduce the complexity, the zonotope that bounds $\Theta$ can be parameterised such that $\mathbf{H}=\lambda \mathbf{H}_{0}$, that corresponds with a zonotope with predefined shape (determined by $\left.\mathbf{H}_{0}\right)$ and a scalar $\lambda$. Then, in this case, the interval prediction thickness (12) is given by

$$
\sum_{k=1}^{M}(\overline{\hat{y}}(k)-\underline{\hat{y}}(k))=2|\lambda| \sum_{k=1}^{M}\left\|\boldsymbol{\varphi}(k) \mathbf{H}_{0}\right\|_{1}=f(|\lambda|)
$$

and restrictions can be expressed as follows:

$$
\lambda\left\|\boldsymbol{\varphi}(k) \mathbf{H}_{0}\right\|_{1} \geq\left|y(k)-\hat{y}^{0}(k)\right|-\sigma \Rightarrow \lambda \geq \frac{\left|y(k)-\hat{y}^{0}(k)\right|-\sigma}{\left\|\varphi(k) \mathbf{H}_{0}\right\|_{1}}
$$

such that Problem 1 can be rewritten as the Problem 2.

\section{Problem 2: "Worst-case Parameter Estimation" (particular case)}

$$
\begin{aligned}
& \min _{\lambda} 2|\lambda| \sum_{k=1}^{N}\left\|\boldsymbol{\varphi}(k) \mathbf{H}_{0}\right\|_{1} \\
& \text { subject to: } \quad \lambda \geq \frac{\left|y(k)-\hat{y}^{0}(k)\right|-\sigma}{\left\|\varphi(k) \mathbf{H}_{0}\right\|_{1}} \quad \forall k=1, \ldots, M
\end{aligned}
$$

The optimal solution of this problem is given by:

$$
\lambda=\sup _{k \in\{1, \ldots, M\}}\left(\frac{\left|y(k)-\hat{y}^{0}(k)\right|-\sigma}{\left\|\boldsymbol{\varphi}(k) \mathbf{H}_{0}\right\|_{1}}\right)
$$

Remark: From Eq. (14) follows: If $\lambda>0, \lambda$ defines a parameter set $\boldsymbol{\Theta}$ parameterised as the zonotope in (2) with $\mathbf{H}=\lambda \mathbf{H}_{0}$ that fulfils conditions (6) for all available data. On the other hand, if $\lambda \leq 0$ then $\hat{y}^{0}(k)-y(k)-\sigma \leq 0$ and $y(k)-\hat{y}^{0}(k)-\sigma$ $\leq 0 \quad \forall k=1, \ldots, M$. This means $y(k) \in\left[\hat{y}^{0}(k)-\sigma, \hat{y}^{0}(k)+\sigma\right] \forall k=1, \ldots, M$. i.e. the only source of uncertainty is the sensor additive noise. 


\subsection{Problem definition}

As in the worst-case approach (section 3), the aim is to estimate parameters and their uncertainty of a model parameterised as in Eq. (1), Given a sequence of data in a fault free scenario and rich enough from the identifiability point of view, that can describe all the measurements $y(k)$ considering now the set-membership approach, this is no variation in parameters.

In this case, the model is called a "consistent model" since the predicted behaviour is always inside the interval of possible measurements. That is $\hat{y}(k) \in[y(k)-\sigma, y(k)+\sigma]$ or alternatively, it can be rewritten as follows

$$
\hat{y}(k) \geq y(k)-\sigma \quad \text { and } \quad \hat{y}(k) \leq y(k)+\sigma \quad \forall k=1, \ldots, M
$$

Where

$$
\hat{y}(k)=\boldsymbol{\varphi}(k) \boldsymbol{\theta} \text { and } \boldsymbol{\theta} \in \boldsymbol{\Theta}
$$

As $\forall \boldsymbol{\theta} \in \boldsymbol{\Theta}$ conditions (15) are satisfied and $\hat{y}(k)$ can be bounded by $\underline{\hat{y}}(k) \leq \hat{y}(k) \leq \overline{\hat{y}}(k)$ where $\underline{\hat{y}}(k)$ and $\overline{\hat{y}}(k)$ are defined as in (7), set-membership conditions (15) can be rewritten as

$$
\underline{\hat{y}}(k) \geq y(k)-\sigma \text { and } \quad \overline{\hat{y}}(k) \leq y(k)+\sigma \quad \forall k=1, \ldots, M
$$

In this case, the model is called a "consistent model" since the predicted behaviour is always inside the interval of possible measurements.

At every instant $k$ the regressor vector $\boldsymbol{\varphi}(k)$ and the measured output $y(k)$ with the set-membership conditions (15) define a strip

$$
\mathbf{F}_{k}=\left\{\boldsymbol{\theta} \in \mathbb{R}^{n_{\theta}}:-\sigma \leq y(k)-\boldsymbol{\varphi}(k) \boldsymbol{\theta} \leq \sigma\right\}
$$

Finally, a set $\boldsymbol{\Theta}$ that fulfils both set-membership conditions (15) $\forall k=1, \ldots, M$ satisfies $\boldsymbol{\Theta} \subseteq\left(\bigcap_{k=1}^{M} \mathbf{F}_{k}\right)$. The following two figures show graphically one example with $n_{\theta}=2$. 

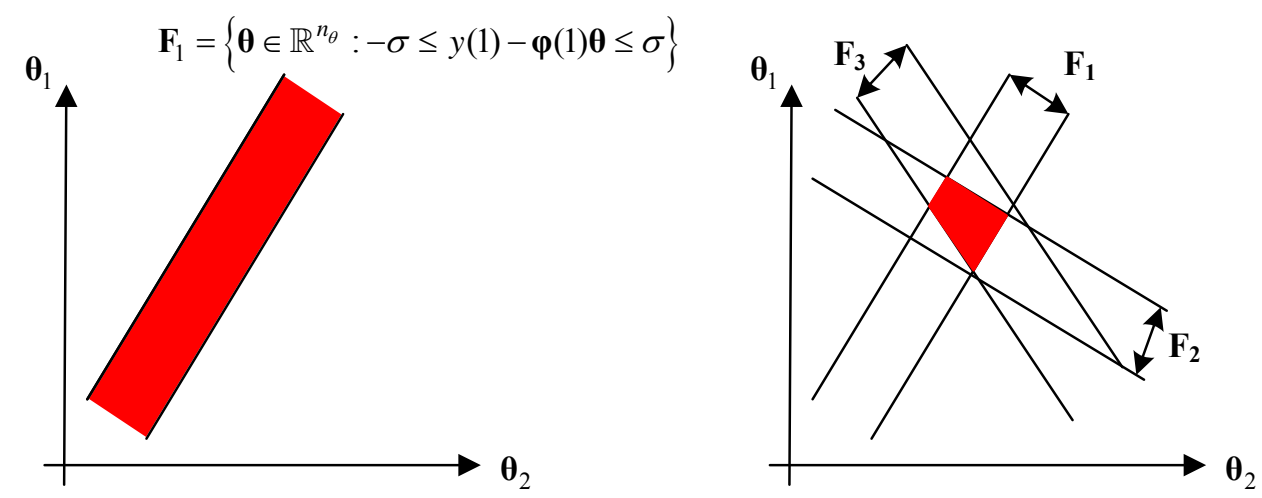

Figure 2. a) Strip with regressor vector and $\varphi(k)$ and measured output $y(k)$ for $k=1$. b) Intersection of the three stripes with data from $k=1$ to $k=3$.

\subsection{Set-Membership parameter estimation}

Using this approach, the parameter set $\boldsymbol{\Theta}$ that contains all models consistent with data, known as Feasible Parameter Set (FPS), is defined as follows:

$$
\mathbf{F P S}=\left\{\boldsymbol{\theta} \in \mathbb{R}^{n_{\theta}} \mid y(k)-\sigma \leq \boldsymbol{\varphi}(k) \boldsymbol{\theta} \leq y(k)+\sigma, k=1, \cdots, M\right\}
$$

The exact description of FPS is in general not simple, and existing algorithms usually approximate the FPS using an inner/outer simpler shapes as boxes, parallelotopes, ellipsoids or zonotopes (Milanese 1996). The approximation set is called Approximated Feasible Parameter Set (AFPS ). In this paper, algorithms that provided inner/outer AFPS using zonotopes in case of using model (1) are used.

\section{Outer approximations}

Outer approximation algorithms find the parameter set $\boldsymbol{\Theta}$ of minimum volume such that $\mathbf{F P S} \subseteq \mathbf{\Theta}$. This kind of algorithms usually implies an excessive computational cost and recursive forms have been proposed in Vicino and Zappa (1996), in case of using parallelotopes, and in Bravo et al. (2006), in case of using zonotopes. These recursive approaches are based in computing iteratively the outer AFPS using parallelotopes/zonotopes and related operations as follows

$$
\begin{gathered}
\mathbf{A F P S}_{2} \supseteq \mathbf{A F P S}_{1} \cap \mathbf{F}_{1} \\
\vdots \\
\mathbf{A F P S}_{k+1} \supseteq \mathbf{A F P S}_{k} \cap \mathbf{F}_{k} \\
\vdots \\
\mathbf{\Theta =} \mathbf{A F P S} \supseteq \mathbf{A F P S}_{M} \cap \mathbf{F}_{M}
\end{gathered}
$$


With $\mathbf{A F P S}$ is the initial set enough large and $\mathbf{F}_{k}$ defined as in (18). For more details in case outer AFPS using zonotopes, see Outer approximation of the Feasible Parameter Set (FPS) using zonotopes in Appendix.

\section{$\underline{\text { Inner approximations }}$}

Inner approximation algorithms find the parameter set $\boldsymbol{\Theta}$ of maximum volume such that $\boldsymbol{\Theta} \subseteq$ FPS .

A set-membership inner approximation using zonotopes parameterised as in Eq (2) for models expressed as in (1) can be obtained in a similar way as proposed to address Problem 2 for the worst-case zonotope. The inner approximation algorithm comes from the fact the FPS conditions (19), considering no variation in parameters, can be bounded by

$$
y(k)-\sigma \leq \underline{\hat{y}}(k) \leq \boldsymbol{\varphi}(k) \boldsymbol{\theta} \leq \overline{\hat{y}}(k) \leq y(k)+\sigma
$$

where $\underline{\hat{y}}(k)$ and $\overline{\hat{y}}(k)$ are defined as in (7) and, in the case of $\boldsymbol{\Theta}$ is a zonotope, calculated as in (9). Then, the maximum inner zonotope, centered in $\boldsymbol{\theta}^{0}$, with consistent parameters can be computed solving the Problem 3.

Problem 3: "Inner Set-membership Zonotope" (general case)

$\max _{\mathbf{H}} f(\boldsymbol{\Theta}(\mathbf{H}))$
subject to: $\|\boldsymbol{\varphi}(k) \mathbf{H}\|_{1} \leq \sigma-\left|y(k)-\hat{y}^{0}(k)\right| \quad \forall k=1, \ldots, M$

In this case, the cost function $f$ is the volume of the zonotope defined by (2). This volume only depends on matrix $\mathbf{H}$ and of $\mathbf{B}^{n}$ with a volume equal to $2^{n}$. In the particular case, $\mathbf{H}$ is a square matrix $\left(n_{\theta}=n\right)$ : $\operatorname{vol}(\boldsymbol{\Theta})=2^{n}|\operatorname{det}(\mathbf{H})|$. See $($ Montgomery, 1989) for more details.

As in Problem 1, the particular case $\mathbf{H}=\lambda \mathbf{H}_{0}$ it will be considered. Then, $\operatorname{vol}(\boldsymbol{\Theta})=f(|\lambda|)^{\ddagger}$ and restrictions of Problem 3 can be expressed as:

$$
\lambda\left\|\boldsymbol{\varphi}(k) \mathbf{H}_{0}\right\|_{1} \leq \sigma-\left|y(k)-\hat{y}^{0}(k)\right| \Rightarrow \lambda \leq \frac{\sigma-\left|y(k)-\hat{y}^{0}(k)\right|}{\left\|\varphi(k) \mathbf{H}_{0}\right\|_{1}}
$$

such that it can be rewritten as follows

\footnotetext{
${ }^{\ddagger}$ For example if $\mathbf{H}_{0}$ is a square matrix $\operatorname{vol}(\boldsymbol{\Theta})=|2 \lambda|^{n}\left|\operatorname{det}\left(\mathbf{H}_{0}\right)\right|$
} 


$$
\begin{aligned}
& \max _{\lambda} \operatorname{vol}(\boldsymbol{\Theta})=f(|\lambda|) \\
& \text { subject to: } \quad \lambda \leq \frac{\sigma-\left|y(k)-\hat{y}^{0}(k)\right|}{\left\|\varphi(k) \mathbf{H}_{0}\right\|_{1}} \quad \forall k=1, \ldots, M
\end{aligned}
$$

The optimal solution of this problem is given by:

$$
\lambda=\inf _{k \in\{1, \ldots, M\}}\left(\frac{\sigma-\left|y(k)-\hat{y}^{0}(k)\right|}{\left\|\boldsymbol{\varphi}(k) \mathbf{H}_{0}\right\|_{1}}\right)
$$

Remark: From Eq. (23) follows: If $\lambda>0, \lambda$ defines a parameter set $\Theta$ parameterised as the zonotope in (2) with $\mathbf{H}=\lambda \mathbf{H}_{0}$ with consistent parameters according to (19). While, if $\lambda=0$, only nominal model is consistent with all data measurements. Then $\boldsymbol{\Theta}=\boldsymbol{\theta}^{0}$. Finally, if $\lambda<0$, then $\boldsymbol{\Theta}=\{\varnothing\}$.

\section{GENERAL CONSIDERATIONS}

\subsection{Nominal parameters $\boldsymbol{\theta}^{0}$ and uncertainty shape $\mathbf{H}_{0}$}

In order to solve Problems 1-4 a nominal estimation $\hat{y}^{0}(k)$ given by the nominal parameters $\boldsymbol{\theta}^{0}$ is needed. These parameters can be obtained in different ways as conventional identification methods (least squares method, for example), using the physical knowledge of the system or using the central point of the outer set-membership approximations (Milanese, 1996 and Ploix 1999).

On the other hand, in order to transform general Problems 1 and 3 (very difficult to solve) to Problems 2 and 4 (easy to solve) it is necessary to determine a matrix $\mathbf{H}_{0}$ that determines the uncertainty shape. This matrix can be given by the covariance of 
parameters calculated using conventional identification methods (least squares method, for example that determines a square covariance matrix), using the physical knowledge of the system, by the method proposed by Bhattacharyya (1995) (diagonal square matrix) or by the zonotope obtained in outer set-membership approximations.

\subsection{Worst-case versus set-membership}

In order to compare both approaches, now we will consider model (1) with parameters $\boldsymbol{\theta}$ belonging to the set $\boldsymbol{\Theta}$ but considering different bounds of additive noise $\sigma_{w c}$ and $\sigma_{s m}$ for the worst-case and set-membership approaches, respectively. The worst-case and set-membership conditions given by (6) and (17) are equivalent when noise bounds satisfy (see Proposition 2 in Appendix)

$$
\sigma_{s m}=2\|\boldsymbol{\varphi}(k) \mathbf{H}\|_{1}+\sigma_{w c}
$$

This condition comes due to fact that worst-case approach considers intrinsically variation in parameters while the setmembership approach does not consider such variation. Thus, the bound additive noise $\sigma_{s m}$ needs an "extra" term $\left(2\|\boldsymbol{\varphi}(k) \mathbf{H}\|_{1}\right)$ that takes into account this variation.

In case of $\sigma_{c s}=\sigma_{w c}$, as was considered before, then $\mathbf{H}=0$ meaning that the nominal model $\boldsymbol{\theta}^{0}$ with additive noise describes the measurements in both approaches: worst-case and set-membership.

But in case of variation in parameters $(\mathbf{H} \neq 0)$, set-membership algorithms (inner and outer) an extra bound should be taken into account additionally to the additive noise.

These conclusions can be extracted from worst-case and set-membership uncertain parameter sets $\left(\boldsymbol{\Theta}^{w c}\right.$ and $\left.\Theta^{s m}\right)$ obtained using Problems 2 and 4 and considering the same additive noise bound $\sigma$.

Given a sequence of $M$ regressor vector values $\varphi(k)$ in a fault free scenario and a model parameterised as in Eq. (1), if

Problem 2 provides $\lambda_{w c}>0$ (obtained using Eq (14)) implying that $\left|y(k)-\hat{y}^{0}(k)\right|-\sigma>0$ for some $k \in\{1, \ldots, M\}$. Then, Problem 4 would provide $\lambda_{s m}<0$ (obtained in Eq. (23)) meaning that it does not exist any model consistent with all available 
data. This result can be explained because in the set of considered data, not only additive noise is present but also parametric uncertainty.

On the other hand, if Problem 4 provides $\lambda_{s m}>0$ (obtained in Eq. (23)) implying $\sigma-\left|y(k)-\hat{y}^{0}(k)\right|>0$ for all $k \in\{1, \ldots, M\}$. Then, Problem 4 would provide $\lambda_{w c}<0$ (obtained using Eq. (14)), meaning that the collected data is described by nominal model plus noise without parameter uncertainty.

Finally, if Problem 2 provides $\lambda_{w c}=0$ (obtained using Eq. (14)), then: $\sup _{k \in\{1, \ldots, M\}}\left(\left|y(k)-\hat{y}^{0}(k)\right|-\sigma\right)=0$. This implies $\lambda_{s m}=0$ (obtained in Eq. (23)). Thus, worst-case model and set-membership model are equal to the nominal model $\boldsymbol{\theta}^{0}$.

\section{FAULT DETECTION TESTS ASSOCIATED TO THE FAULT IDENTIFICATION APPROACHES}

According to Guerra (2008), fault detection, considering the model (1) and the parameter uncertainty set parameterised as in (2), can be either based on a "direct" or "inverse" test. These two tests are associated to each of the two parameter estimation approaches presented in Sections 3 and 4: the direct test with the worst-case approach while the inverse test with the Invariant approach.

\subsection{Direct test}

The direct test is based on the evaluation of the residual obtained from difference between measurements and model prediction at every instant $k$

$$
r(k)=y(k)-\hat{y}(k)=y(k)-\varphi(k) \boldsymbol{\theta}(k)
$$

Ideally, the residual given by Eq (25), known as parity equation (Iserman, 2006), in case that neither modelling errors nor noise were present, it should be different from zero in a faulty scenario and zero otherwise. However, because of modelling errors, the detection test is equivalent to check the following condition assuming parametric uncertainty

$$
0 \notin \Gamma(k)
$$

where $\Gamma(k)$ is the set of possible residuals according to the parameter uncertainties and the additive noise.

$$
\Gamma(k)=\{r(k) \mid r(k)=y(k)-\varphi(k) \boldsymbol{\theta}(k)-e(k) ; \boldsymbol{\theta}(k) \in \boldsymbol{\Theta} \text { and }|e(k)| \leq \sigma\}
$$

Or, alternatively, to check if

$$
y(k) \notin \Upsilon(k)
$$


where $\Upsilon(k)$ is the direct image of the uncertain model defined as

$$
\Upsilon(k)=\{\hat{y}(k)+e(k) \mid \hat{y}(k)=\boldsymbol{\varphi}(k) \boldsymbol{\theta}(k), \boldsymbol{\theta}(k) \in \boldsymbol{\Theta} \text { and }|e(k)| \leq \sigma\}=[\underline{\hat{y}}(k)-\sigma, \overline{\hat{y}}(k)+\sigma]
$$

Remark: In the evaluation of possible residuals $\Gamma(k)$ and the direct image not consideration of $\boldsymbol{\theta}(k-1)$ is assumed, then no restriction in variation from parameter $\boldsymbol{\theta}(k-1)$ to parameter $\boldsymbol{\theta}(k)$ is assumed.

\subsection{Inverse test}

The inverse test consists on checking at every instant $k$ if there not exist any parameter in the parameter uncertainty set $\boldsymbol{\Theta}$ such that model (1) is consistent with all the system measurements.

$$
\neg \exists \boldsymbol{\theta} \in \boldsymbol{\Theta} \mid y(n)-\sigma \leq \boldsymbol{\varphi}(n) \boldsymbol{\theta} \leq y(n)+\sigma \quad \forall n=1, \ldots, k
$$

This is the same that check if

$$
\mathbf{F P S}_{k} \cap \mathbf{F}_{k}=\varnothing
$$

where $\mathbf{F P S}_{k}$ is the Feasible parameter set consistent with all the previous measurements at instant $k$ and belonging to $\boldsymbol{\Theta}$

$$
\mathbf{F P S}_{k}=\{\boldsymbol{\theta} \in \boldsymbol{\Theta} \mid y(i)-\sigma \leq \boldsymbol{\varphi}(i) \boldsymbol{\theta} \leq y(i)+\sigma, i=1, \cdots, k-1\}
$$

and $\mathbf{F}_{k}$ is the strip of parameters consistent with measurement $k$ defined in (18)
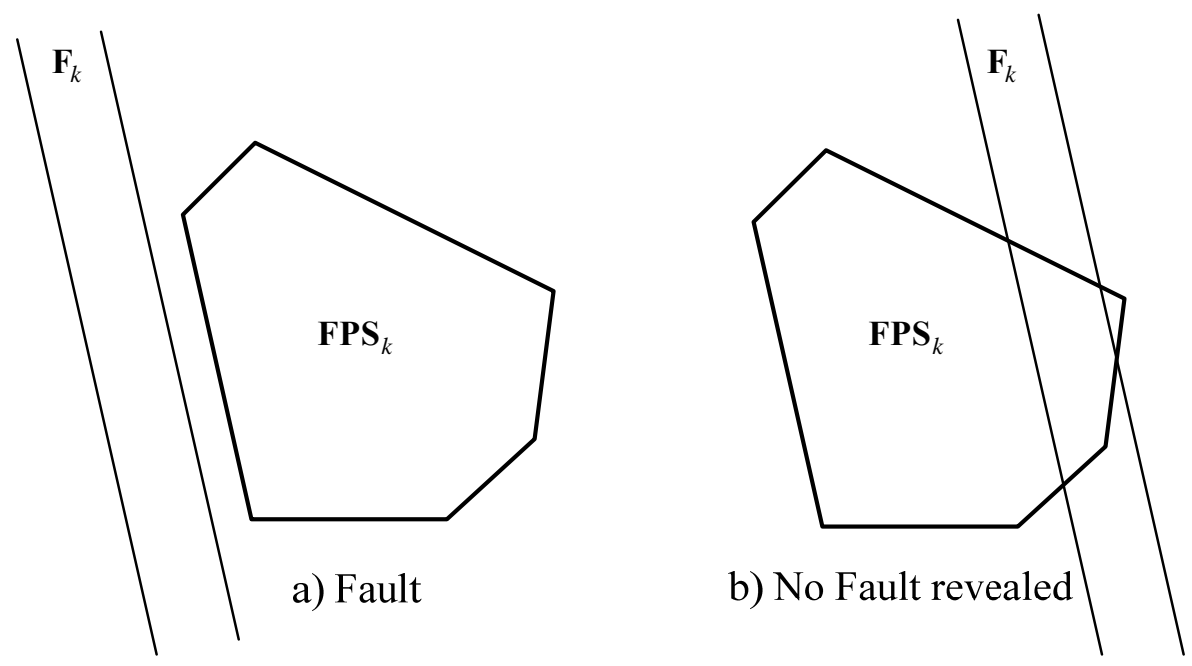

Figure 3. Fault and No Fault revealed situations using the FPS 
In case of using approximations of the FPS, for instance outer zonotopes, as was described on section 4.2, this test can be easily implemented using the recursively parameter estimation procedure (20) but considering an initial set

$$
\mathbf{A F P S}_{1}=\Theta
$$

Then (31) leads to

$$
\mathbf{A F P S}_{k+1}=\mathbf{A F P S}_{k} \cap \mathbf{F}_{k}=\varnothing
$$

When using in (34) an outer approximation of FPS, denoted as $\mathbf{A}_{\text {out }} \mathbf{F P S}$, a missed alarm could appear when $\mathbf{A}_{\text {out }} \mathbf{F P S}_{k+1} \neq \varnothing$ is obtained since $\mathbf{A}_{\text {out }}$ FPS contains parameters that do not belong to FPS (Fig 4 c). On the other hand, if an inner approximation $\mathbf{A}_{\text {in }}$ FPS is used in (34), a false alarm might appear when $\mathbf{A}_{\text {in }} \mathbf{F P S} \mathbf{S}_{k+1}=\varnothing$ is obtained since $\mathbf{A}_{\text {in }}$ FPS does not contains all the parameters contained in FPS (Fig 4 d). Thus, in spite of the outer approximation $\mathbf{A}_{\text {out }}$ FPS is preferable in fault detection since avoid false alarms, the inner approximation $\mathbf{A}_{i n}$ FPS can provide complementary information to the fault detection since the cases presented in Figure 4 can be distinguished. Moreover, knowing both approximations the size of region in between them can give an idea of the precision of the FPS approximation. 

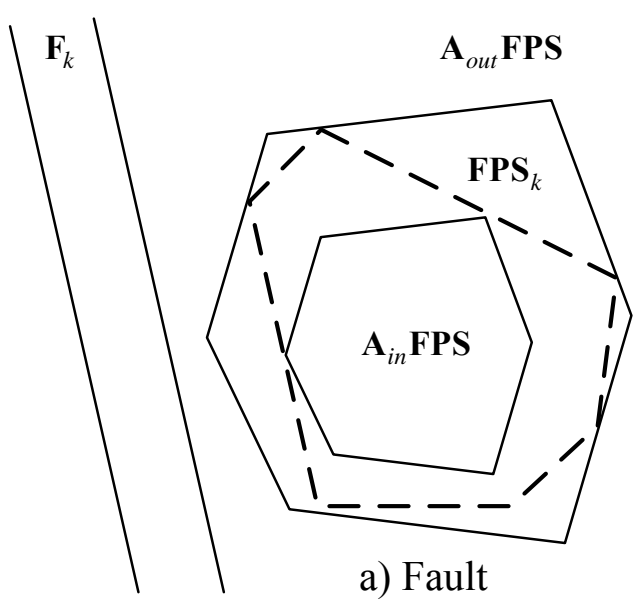

a) Fault

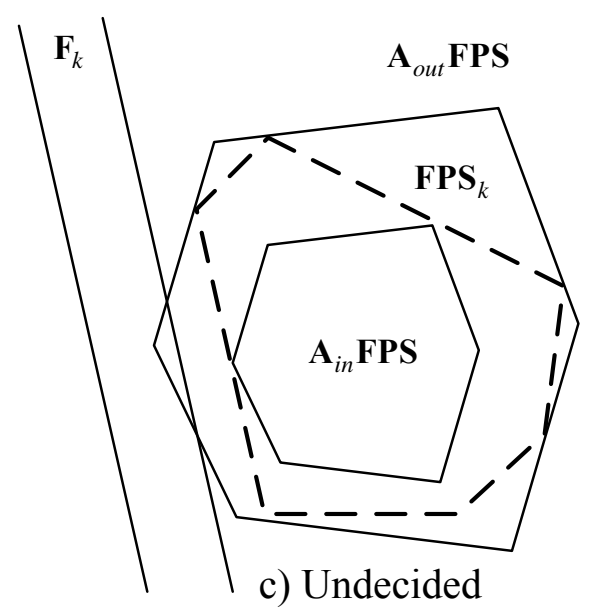

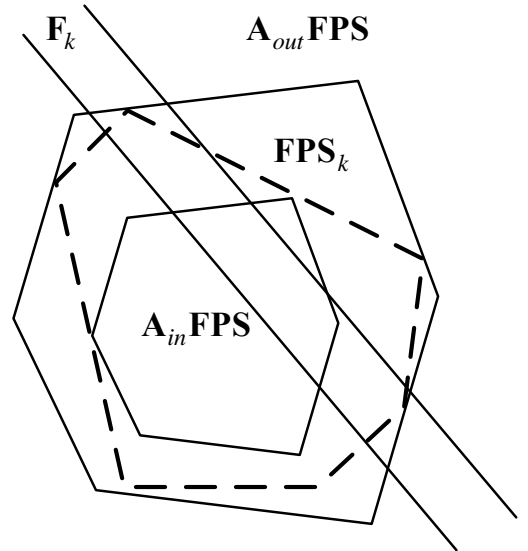

b) No Fault revealed

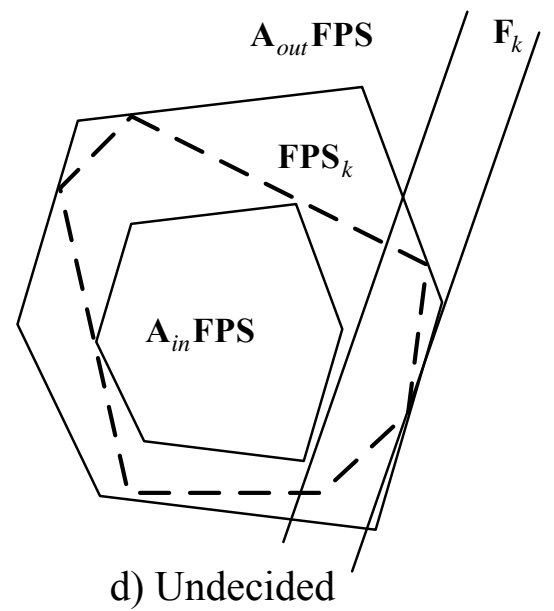

Figure 4. Different situations that can be distinguished in fault detection when using inner and outer approximations

Another way to understand the inverse test is to consider it as a parameter estimation (Puig, 2006), where for every time instant $k$, the parameter vector $\boldsymbol{\theta}(k)$ can be estimated from input and output measurements over a $\mathrm{N}$ samples temporal window as follows

$$
\begin{gathered}
\hat{\boldsymbol{\theta}}(k)=\boldsymbol{g}(\Phi(k)) \boldsymbol{Y}(k)^{\S} \\
\text { where } \Phi(k)=\left(\begin{array}{c}
\boldsymbol{\varphi}(k-N) \\
\vdots \\
\boldsymbol{\varphi}(k)
\end{array}\right) \text { and } \boldsymbol{Y}(k)=\left(\begin{array}{c}
y(k-N) \\
\vdots \\
y(k)
\end{array}\right)
\end{gathered}
$$

Then, the inverse test, in a similar way than the direct test, is based on evaluating the following residual (Gertler, 1998) 


$$
\boldsymbol{r}_{\boldsymbol{\theta}}(k)=\boldsymbol{g}(\boldsymbol{\Phi}(k)) \boldsymbol{R}(k)=\boldsymbol{g}(\Phi(k))(\boldsymbol{Y}(k)-\hat{\boldsymbol{Y}}(k))=\hat{\boldsymbol{\theta}}(k)-\boldsymbol{\theta}(k)
$$

with $\boldsymbol{R}(k)=\left(\begin{array}{c}r(k-N) \\ \vdots \\ r(k)\end{array}\right)$ and where $\boldsymbol{\theta}(k)$ is the normal value obtained from system modelling in a non-faulty situation. This, the detection test (30) can be rewritten as follows

$$
0 \notin \Gamma_{\theta}(k)
$$

where

$$
\Gamma_{\boldsymbol{\theta}}(k)=\left\{\boldsymbol{r}_{\boldsymbol{\theta}}(k) \mid \boldsymbol{r}_{\boldsymbol{\theta}}(k)=\hat{\boldsymbol{\theta}}(k)-\boldsymbol{\theta}(k), \boldsymbol{\theta}(k) \in \mathbf{A F P S}_{k}\right\}
$$

Remark: The implementation of (37) instead of (30), that always can be implemented, is subject to the condition of permanent excitation $(\Phi(k)$ is invertible).

\section{FAULT SENSITIVITY OF THE PROPOSED FAULT DETECTION TESTS}

In fault detection, two kinds of faults are typically considered: additive faults (in input/output variables) and multiplicative faults (in parameters) (Gertler, 1998). Thus, including both type of faults in the system (1), the residual can be written as

$$
y(k)=\boldsymbol{\varphi}(k) \boldsymbol{\theta}(k)+f_{\varphi}(k) \boldsymbol{\theta}(k)+f_{y}(k)+\boldsymbol{\varphi}(k) f_{\boldsymbol{\theta}}(k)+e(k)
$$

where $f_{\varphi}(k)$ and $f_{y}(k)$ represent the input and output sensor faults (additive) and $f_{\theta}(k)$ represent the parametric faults (multiplicative).

Eq. (1) can be rewritten as

$$
y(k)=y_{0}(k)+\delta_{f}(k)+e(k)
$$

where $y_{0}(k)$ is the non-fault response and $\delta_{f}(k)$ is the effect of the faults

$$
\begin{gathered}
y_{0}(k)=\boldsymbol{\varphi}(k) \boldsymbol{\theta}(k) \\
\delta_{f}(k)=f_{\varphi}(k) \boldsymbol{\theta}(k)+f_{y}(k)+\boldsymbol{\varphi}(k) f_{\boldsymbol{\theta}}(k)
\end{gathered}
$$

\footnotetext{
${ }^{\S}$ In the case of a linear in the parameters models, Eq. (30) corresponds to the non-recursive least squares formula: $\boldsymbol{g}(\Phi(k))=\left(\Phi^{t}(k) \Phi(k)\right)^{-1} \Phi^{t}(k)$
} 
According to Gertler (1998), the minimum detectable fault corresponds to a fault that brings a residual to its threshold (“triggering limit”), assuming that no other faults and nuisance inputs are present.

In this section it will be determined the minimum effect of a fault $\delta_{f}(k)$ that will guarantee the fault detection in the fault detection methods presented in section 6 .

\section{$\underline{\text { Direct test }}$}

In case of using model (1), the set of possible residual defined in (27) can be bounded by

$$
\Gamma(k)=[\underline{r}(k), \bar{r}(k)]
$$

where $\underline{r}(k)=y(k)-\overline{\hat{y}}(k)-\sigma$ and $\bar{r}(k)=y(k)-\underline{\hat{y}}(k)+\sigma$, with $\overline{\hat{y}}(k)$ and $\underline{\hat{y}}(k)$ as defined in (7), then test (26) detects an inconsistency when

$$
\underline{r}(k)>0 \text { or } \bar{r}(k)<0
$$

that, considering (40), implies

$$
\begin{aligned}
& \delta_{f}(k)>\overline{\hat{y}}(k)+\sigma-y_{0}(k)-e(k) \\
& \delta_{f}(k)<\underline{\hat{y}}(k)-\sigma-y_{0}(k)-e(k)
\end{aligned}
$$

In order to bound the inequalities the smallest values of $y_{0}(k)$ and $e(k)$ are considered in (45a) and the biggest values in (45b), then

$$
\delta_{f}(k)>\overline{\hat{y}}(k)-\underline{\hat{y}}(k)+2 \sigma \text { and } \quad \delta_{f}(k)<\underline{\hat{y}}(k)-\overline{\hat{y}}(k)-2 \sigma
$$

This is

$$
\left|\delta_{f}(k)\right|>\overline{\hat{y}}(k)-\underline{\hat{y}}(k)+2 \sigma
$$

That in the case of bounding the parameters by a Zonotope leads to

$$
\left|\delta_{f}(k)\right|>2\|\boldsymbol{\varphi}(k) \mathbf{H}\|_{1}+2 \sigma
$$




\section{$\underline{\text { Inverse test }}$}

In case of using model (1), and the test based on (34) where the Aproximation of the feasible parameter set at instant $k$ can be bounded by a zonotope as

$$
\mathbf{A F P S}_{k}=\boldsymbol{\theta}_{k}^{0} \oplus \boldsymbol{X}_{k} \mathbf{B}^{n}
$$

and the strip $\mathbf{F}_{k}$ as defined in (18) can be implemented using the zonotope support strip (Bravo 2006) defined by

$$
\mathbf{F}_{k}=\left\{\boldsymbol{\theta} \in \mathbb{R}^{n_{\theta}}: q_{d} \leq \boldsymbol{\varphi}(k) \boldsymbol{\theta} \leq q_{u}\right\}
$$

where

$$
\begin{gathered}
q_{u}=\boldsymbol{\varphi}(k) \boldsymbol{\theta}_{k}^{0}+\left\|\boldsymbol{\varphi}(k) \boldsymbol{X}_{k}\right\|_{1} \\
q_{d}=\boldsymbol{\varphi}(k) \boldsymbol{\theta}_{k}^{0}-\left\|\boldsymbol{\varphi}(k) \boldsymbol{X}_{k}\right\|_{1}
\end{gathered}
$$

Then the test (34) is equivalent to check if

$$
q_{d}>y(k)+\sigma \text { or } q_{u}<y(k)-\sigma
$$

For more details see Vicino \& Zappa (1996).

Inequalities (50) taking into account (40) lead to

$$
\begin{array}{r}
\delta_{f}(k)<q_{d}-\sigma-y_{0}(k)-e(k) \\
\delta_{f}(k)>q_{u}+\sigma-y_{0}(k)+e(k)
\end{array}
$$

In order to bound the inequalities the biggest values of $y_{0}(k)$ and $e(k)$ are considered in (52a) and the smallest values in (52b), then

$$
\delta_{f}(k)>2\left\|\boldsymbol{\varphi}(k) \boldsymbol{X}_{k}\right\|_{1}+2 \sigma \text { and } \quad \delta_{f}(k)<-2\left\|\boldsymbol{\varphi}(k) \boldsymbol{X}_{k}\right\|_{1}-2 \sigma
$$

This is

$$
\left|\delta_{f}(k)\right|>2\left\|\boldsymbol{\varphi}(k) \boldsymbol{X}_{k}\right\|_{1}+2 \sigma
$$

As can be extracted from (47) and (53) the minimum effect detectable of a fault have the same structure from the direct and inverse tests.

$$
\left|\delta_{f}(k)\right|>\delta_{r}(\varphi(k))
$$

where $\delta_{r}(k)$ is the interval prediction thickness considering the additive noise. This is

$$
\delta_{r}(k)=\max (\boldsymbol{\varphi}(k) \boldsymbol{\theta}(k)+e(k))-\min (\boldsymbol{\varphi}(k) \boldsymbol{\theta}(k)+e(k))
$$


with $\boldsymbol{\theta}(k) \in \boldsymbol{\Theta}_{k}$ and $|e(k)| \leq \sigma$

the difference is that the set of parameters $\boldsymbol{\Theta}_{k}$ and additive noise may be different.

In this work we will focus only in the instant output sensor faults (additive) and parametric faults (multiplicative).

In case of instant output sensor faults the effect of the fault is

$$
\delta_{f}(k)=f_{y}(k)
$$

while in the case of parametric fault is

$$
\delta_{f}(k)=\varphi(k) f_{\theta}(k)
$$

Remark that the effect of parametric faults depends on the regressor vector.

\section{CASE STUDY: FOUR TANKS SYSTEM}

\subsection{Description of the system}

A quadruple-tank process, proposed by Johansson (2000), will be used to illustrate the results presented in this paper. A schematic diagram of the system is shown in Fig. 4a. The process inputs are $v_{1}$ and $v_{2}$ (input voltages to the pumps) and the outputs are the tank levels $h_{1}$ and $h_{2}$.

The experiments presented in this section just consider the residual coming from the first tank, assuming that levels $h_{1}$ and $h_{3}$ and voltage $v_{1}$ are measured:

$$
\frac{d h_{1}}{d t}=-\frac{a_{1}}{A_{1}} \sqrt{2 g h_{1}}+\frac{a_{3}}{A_{1}} \sqrt{2 g h_{3}}+\frac{\gamma_{1} k_{1}}{A_{1}} v_{1}+e_{1}(k)
$$

where $e_{1}(k)$ is the measured noise $\left(\left|e_{1}(k)\right| \leq \sigma=0.05 \mathrm{~cm}\right)$ and $A_{1}=28 \mathrm{~cm}^{2}, k_{1}=3.33 \mathrm{~cm}^{3} / V \mathrm{~s}, g=98 \mathrm{~cm} / \mathrm{s}^{2}$ and $\gamma_{1}=0.7$ assumed constants.

Eq. (45) can be discretized by the Euler method with sampling time $\Delta t=1 \mathrm{~s}$ : 


$$
h_{1}(k)=h_{1}(k-1)-\frac{a_{1}}{A_{1}} \sqrt{2 g h_{1}(k-1)}+\frac{a_{3}}{A_{1}} \sqrt{2 g h_{3}(k-1)}+\frac{\gamma_{1} k_{1}}{A_{1}} v_{1}(k-1)+e_{1}(k)
$$

and can be transformed in a LPV model as

$$
h_{1}(k)=a\left(p_{1}(k)\right) h_{1}(k-1)+b_{1}\left(p_{2}(k)\right) h_{3}(k-1)+b_{2} v_{1}(k-1)+e_{1}(k)
$$

where: $\quad p_{1}(k)=h_{1}(k-1) \quad$ and $\quad p_{2}(k)=h_{3}(k-1) \quad$ are $\quad$ the $\quad$ scheduling $\quad$ variables; $\quad a\left(p_{1}(k)\right)=1-\frac{a_{1}}{A_{1}} \sqrt{\frac{2 g}{p_{1}(k)}} \quad$ and $b_{1}\left(p_{2}(k)\right)=\frac{a_{3}}{A_{1}} \sqrt{\frac{2 g}{p_{2}(k)}}$ are the LPV parameters; $b_{2}=\frac{\gamma_{1} k_{1}}{A_{1}}$ is a LTI parameter.

\subsection{Experiment definition}

In order to apply identification techniques presented in Section 3, a fault free scenario has been recorded (Fig. 4b).

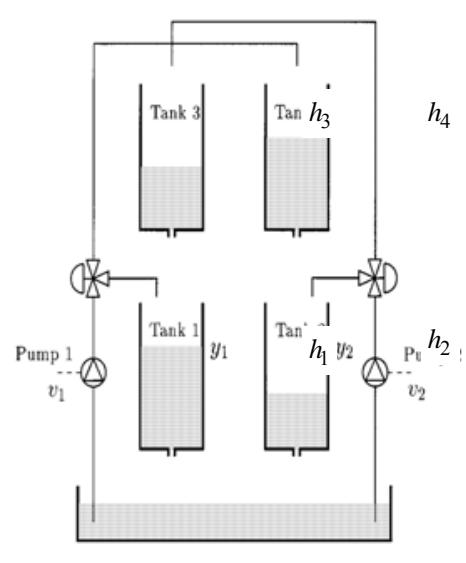

(a)
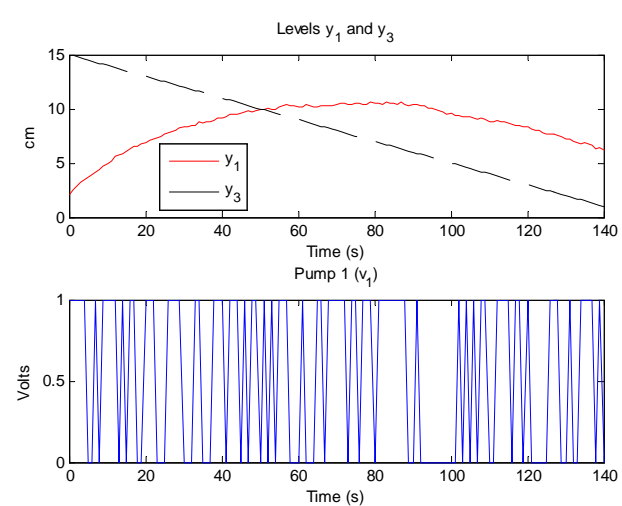

(b)

Figure 4: (a) Quadruple-tank process. (b) Fault free scenario

To illustrate and compare the different behaviour of the two proposed identification methods, two cases will be considered:

Case 1. Exact nominal model based in (47), that can be expressed in regressor form as follows:

$$
y_{1}(k)=\varphi(k) \boldsymbol{\theta}\left(\mathbf{p}_{k}\right)+e(k)
$$


with: $y_{1}(k-1)=h_{1}(k-1), \boldsymbol{\varphi}(k)=\left(\begin{array}{l}y_{1}(k-1) \\ u_{1}(k-1) \\ u_{2}(k-1)\end{array}\right)^{T}$ and $\boldsymbol{\theta}\left(\mathbf{p}_{k}\right)=\left(\begin{array}{c}a\left(p_{1}(k)\right) \\ b_{1}\left(p_{2}(k)\right) \\ b_{2}\end{array}\right)$

where $u_{1}(k-1)=h_{3}(k-1), u_{2}(k-1)=v_{1}(k-1), p_{1}(k)=y_{1}(k-1)$ and $p_{2}(k)=u_{1}(k-1)$.

To apply the methods of identification and fault detection described in Sections 3 and 4 to the LPV model, Eq. (48) can be rewritten as: $\quad y_{1}{ }^{\prime}(k)=\boldsymbol{\varphi}(k) \boldsymbol{\omega}+e(k)=\hat{y}_{1}{ }^{\prime}(k)+e(k) \quad$ with: $\quad y_{1}{ }^{\prime}(k)=y_{1}(k)-\hat{y}_{1}^{0} \quad$ and $\quad \boldsymbol{\omega}=\left(\omega_{1}, \omega_{2}, \omega_{3}\right)^{T} \in \mathbf{\Omega}=\boldsymbol{\omega}_{0} \oplus \mathbf{H B}^{n}$ where $\hat{y}_{1}^{0}(k)=\boldsymbol{\varphi}(k) \boldsymbol{\theta}^{0}\left(\mathbf{p}_{k}\right)$. Nominal LPV parameters come from Eq. (47)

$$
\boldsymbol{\theta}^{0}\left(\mathbf{p}_{k}\right)=\left(\begin{array}{c}
1-\frac{0.1123}{\sqrt{p_{1}(k-1)}} \\
\frac{0.1123}{\sqrt{p_{2}(k-1)}} \\
0.0833
\end{array}\right)
$$

Case 2. Approximate nominal model based on approximating LPV parameters $a\left(p_{1}(k)\right)$ and $b_{1}\left(p_{2}(k)\right)$ coming from Eq. (47) by their mean values: $a=a^{0}=0.9461, b_{1}=b_{1}^{0}=0.0680$.
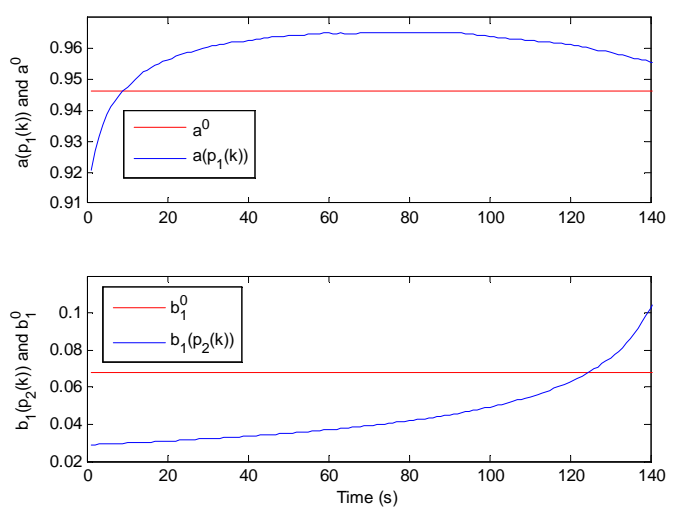

Figure 5: LPV parameter of Case 1 and approximate parameter of Case 2 evolutions in the fault free scenario

Remark: Notice that in Case 1 the only source of uncertainty is the additive noise in the measurements while in Case 2 additionally there is some parametric uncertainty because of the approximation of the LPV parameters by a constant value. 
A worst-case model has been obtained solving Problem 2 using a diagonal square matrix $\mathbf{H}_{0}=\operatorname{diag}\left(\lambda_{1}^{0}, \lambda_{2}^{0}, \lambda_{3}^{0}\right)$ where $\lambda_{1}^{0}=0.0385, \lambda_{2}^{0}=0.0781, \lambda_{3}^{0}=0$ (no uncertainty in parameter $b_{2}$ has been considered) are obtained from the maximum variations in parameters $a\left(p_{1}(k)\right)$ and $b_{1}\left(p_{2}(k)\right)$ in the considered operating range: $y_{1} \in[2.34,10.54] \mathrm{cm}$ and $u_{1} \in[1,15] \mathrm{cm}$.

Case 1 Results. In this case since there is not parametric uncertainty, all the data could be explained by the worst-case model just adding the noise bounds to the predicted output provided by the nominal model. This is confirmed when applying Eq. (14), solution of Problem 2, since $\lambda=-0.0063<0$, implying no uncertainty in parameters is necessary to cover the data with the worst-case prediction as discussed in Section 3.4

Case 2 Results. In this case, the solution of Problem 2 using Eq. (14) leads to $\lambda=0.4523$. Taking into account the considered matrix $\mathbf{H}_{\mathbf{0}}$ obtained at the beginning of this section results in $\mathbf{H}=\lambda \operatorname{diag}\left(\lambda_{1}^{0}, \lambda_{2}^{0}, 0\right)$. This means that the parametric uncertainty corresponding to $a$ and $b_{1}$ is $\pm \lambda \lambda_{1}^{0}$ and $\pm \lambda \lambda_{2}^{0}$, respectively (See Fig. 6a). From Fig. 6b, it can be noticed that all measurements are covered by the bounds of the worst-case prediction.

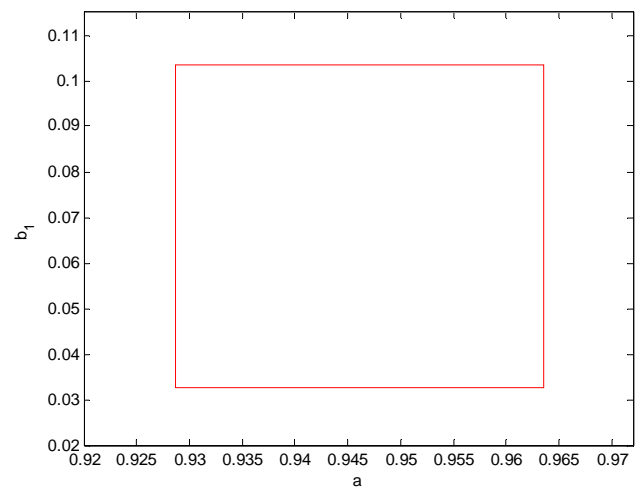

(a)

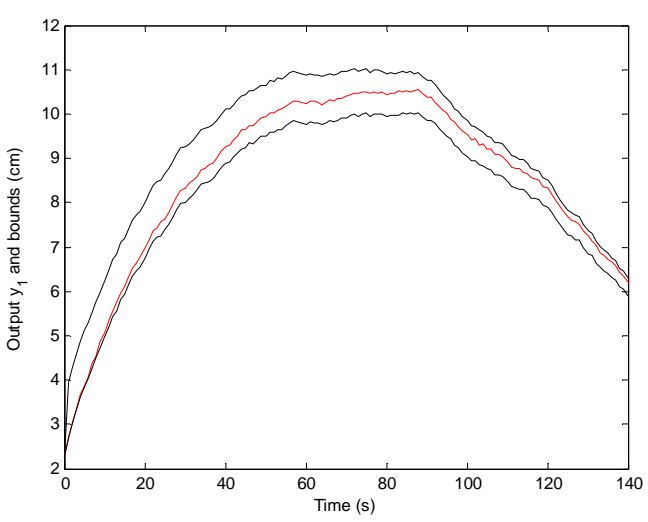

(b)

Figure 6: (a) Uncertain parameters $a$ and $b_{1}$. (b) Measured output and bounds 
Using the set-membership estimation methods proposed in Section 3.2, the FPS has been approximated by an outer zonotope. Additionally, the inner zonotope has been obtained solving Problem 4 with Eq. (20) and using as $\mathbf{H}_{\mathbf{0}}$ the $\mathbf{H}_{\text {matrix of the outer }}$ zonotope.

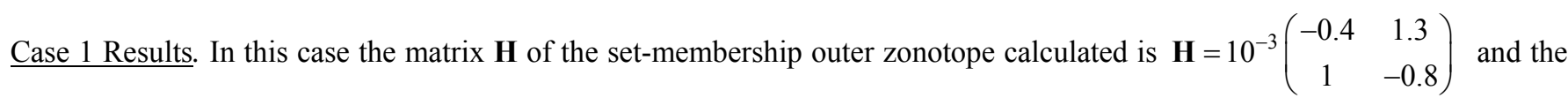
solution of Problem 4 using (14) leads to $\lambda=0.59$. Both inner and outer zonotopes calculated are showed in Fig. 7, where all strips obtained from every measurement are presented.

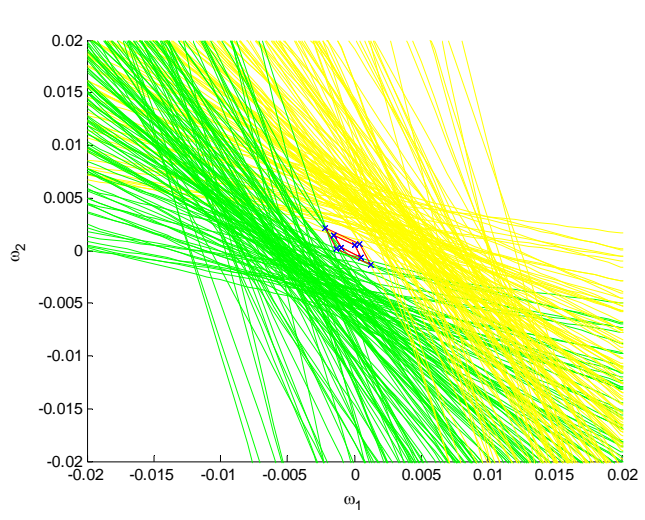

(a)

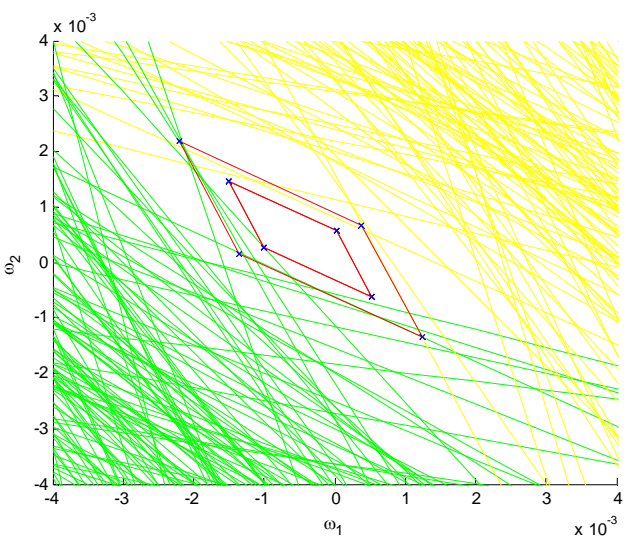

(b)

Figure 7: (a) Set-membership inner and outer zonotopes corresponding to Case 1. (b) Detail

Case 2 Results. In this case since there is parametric uncertainty and the considered set-membership model only considers

additive noise, the FPS is empty. That is $\bigcap_{k=1}^{M} \mathbf{F}_{k}=\varnothing$, implying that does not exist a set-membership model with the assumed structure consistent with all the identification data. But increasing the noise bound $(\sigma=0.05 \mathrm{~cm})$ by the "extra" term $2\|\boldsymbol{\varphi}(k) \mathbf{H}\|_{1}$ (with $\mathbf{H}=0.4523 \operatorname{diag}(0.0385,0.0781,0)$ obtained from the worst-case model corresponding to Case 2) , a setmembership model can be obtained with a FPS equal to the one of the worst-case model presented in Figure 6a.

\subsection{Fault detection results}

In order to show the behaviour of the two tests in front of different faults, two different kinds of faults have been simulated: additive (in sensors) and a multiplicative (in components) faults. 
The model that has been used in the direct test is the identified using the worst-case approach with the approximate nominal model (Case 2) as described in Section 7.3. On the other hand, the model that has been used in the inverse test is the identified using the set-membership approach with nominal LPV model (Case 1) as described in Section 7.4.

Fault scenario 1: "Additive fault in input $u_{1} f_{u_{1}}=12$ Volts at $t=60 \mathrm{~s}$ "

Figure 8 shows the behaviour of the direct test. In this figure, it can be seen that as the measurement at $t=61 \mathrm{~s}$ is out of the bounds, calculated by the worst-case model, the fault is detected at this instant. On the other hand the residual for $t>60 \mathrm{~s}$ tends to a constant value.

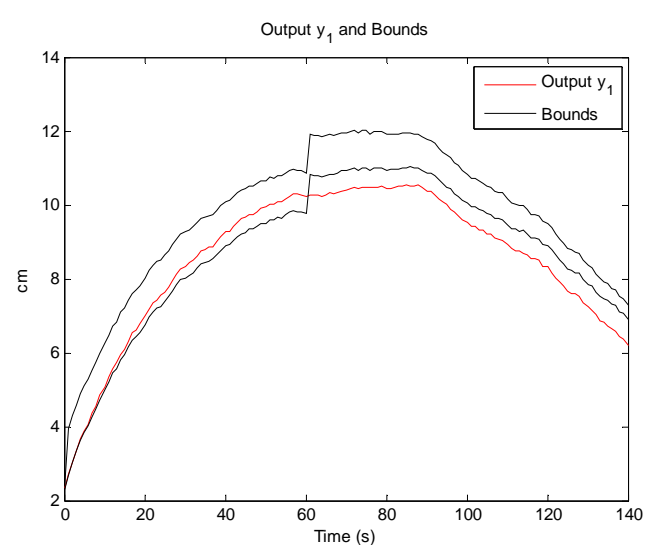

(a)
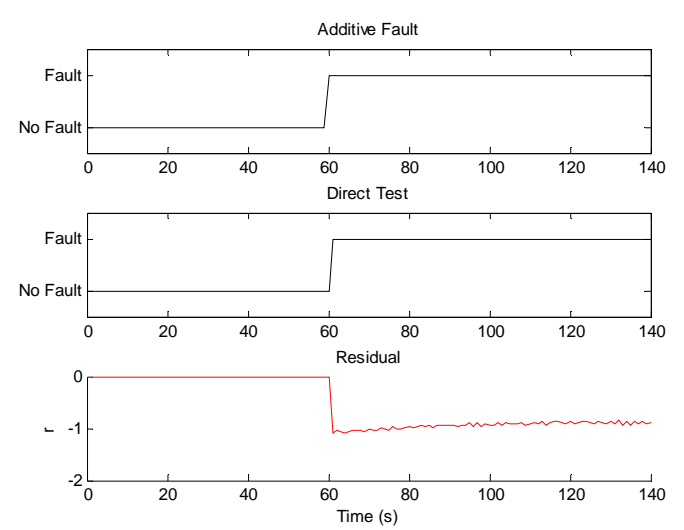

(b)

Figure 8. (a) Measured output and bounds. (b) Direct test Fault detection in fault scenario 1

Figure 9 shows the behaviour of the inverse test in this scenario. In this figure, it can be seen that as the stripe at $t=61 \mathrm{~s}$ does not intersect the outer zonotope, of the set-membership model, the fault is detected at this instant. On the other hand the intersection of the stripes for $t>60 \mathrm{~s}$ is empty and then does not define a new set of parameters consistent with the fault data. This fact can be explained because the fault is additive.
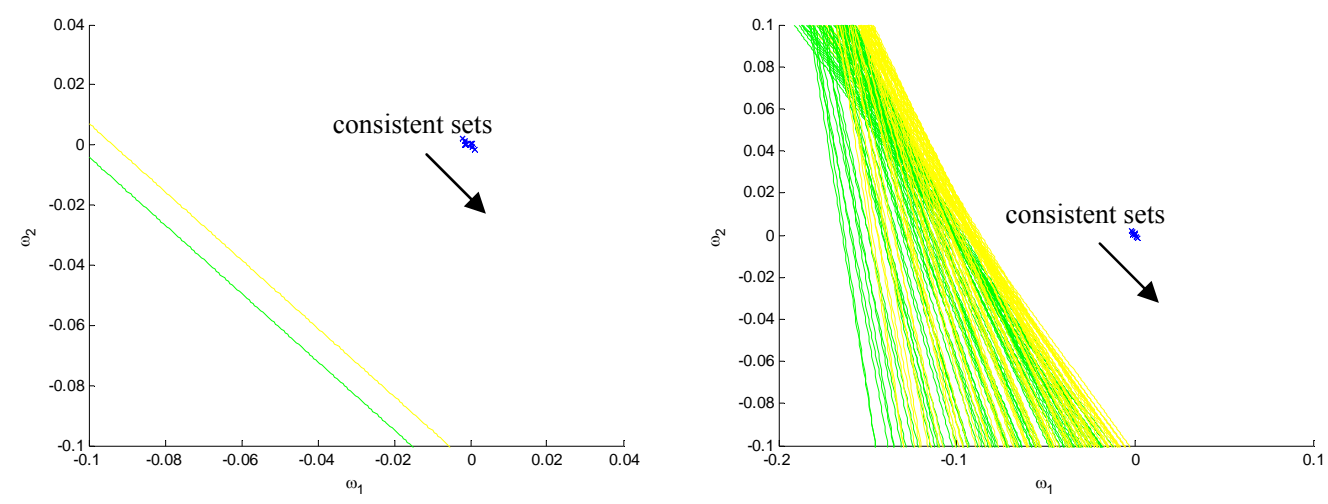
Figure 9. Inverse test fault detection in fault scenario 1: (a) Outer and inner zonotope and hyper-stripe at $t=61 \mathrm{~s}$. (b) Outer and inner zonotope and hyper-stripes for $t>60 \mathrm{~s}$

Fault scenario 2: “Multiplicative Fault 70\% of widening in the exit of tank number 3" (parameter $a_{1}$ in Eq. (46)) at $t=20 \mathrm{~s}$ ”.

Fig. 10 shows the behaviour of the direct test. In this figure, it can be seen that as the measurement is not out of the bounds, calculated by the worst-case model, until at $t=82 \mathrm{~s}$ the fault is not detected until this instant. On the other hand the residual for $t>82 s$ varies.

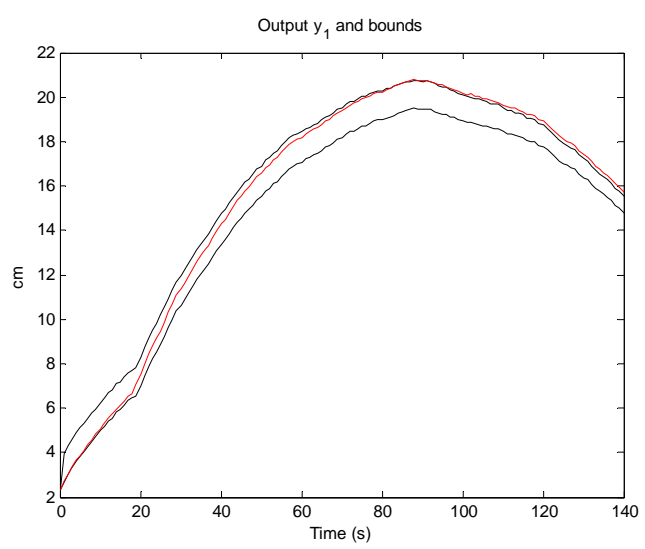

(a)
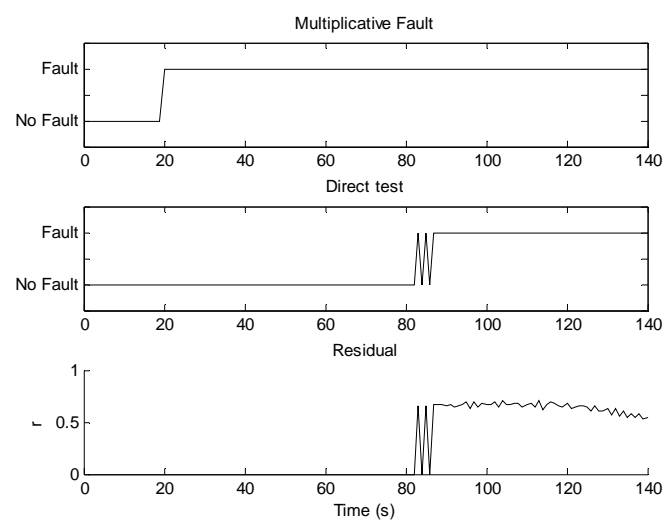

(b)

Figure 10. (a) Measured output and bounds. (b) Direct test Fault detection in fault scenario 2

Figure 11 shows the behaviour of the inverse test in this scenario. In this figure, it can be seen that as the stripe at $t=21 \mathrm{~s}$ does not intersect with the outer zonotope, of the set-membership model, the fault is detected at this instant. On the other hand, the intersection of the stripe for $t>20$ s define a new set of parameter consistent with the fault data that can be approximated by zonotopes. Implying the existence of a parametric fault. 


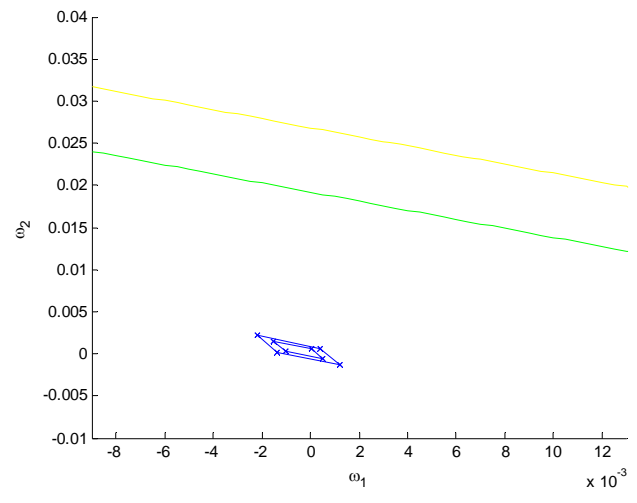

(a)

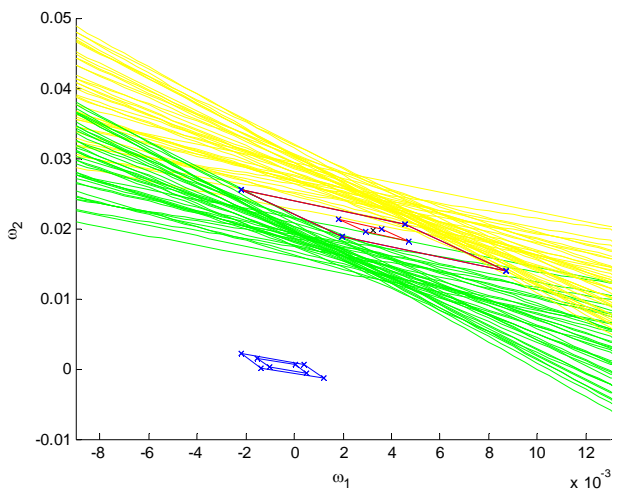

(b)

Figure 11. Inverse test fault detection in fault scenario 2: (a) Outer and inner zonotopes and hyper-stripe at $t=21 \mathrm{~s}$. (b) Outer and inner zonotopes and hyper-stripes for $t>21 s$ with the Outer and inner zonopes consistent with the new data.

\subsection{Sensitivity results}

According to the results obtained in Section 5, the values of sensitivity to additive and multiplicative faults have been obtained for direct and inverse test. This sensitivities have been calculated as the steady-state value of the transfer functions defined by equations (36) and (37) for additive and multiplicative faults in direct test. On the other hand, (38) and (39) for additive and multiplicative faults in inverse test.

These sensitivities are showed in Fig. 12 and 13, where it can be seen that sensitivity of direct test to additive faults is constant as sensitivity of inverse test to multiplicative faults. But sensitivity of direct test to multiplicative faults depends on the operating point as sensitivity of inverse test to additive faults.
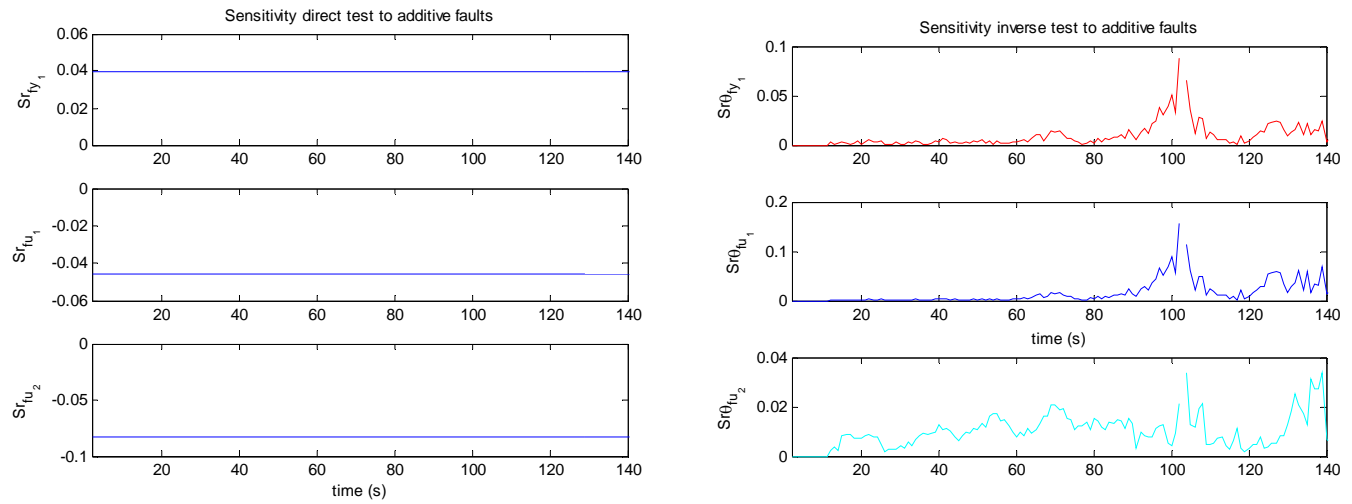

Figure 12. Direct and inverse test fault sensitivity to additive faults 

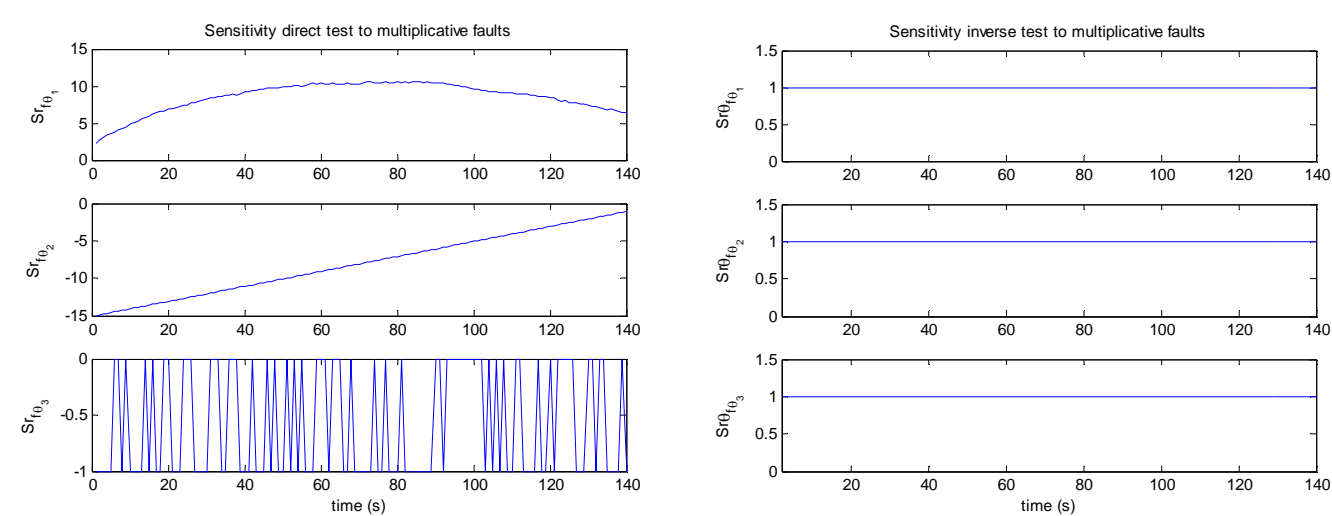

Figure 13. Direct and inverse test fault sensitivity to multiplicative faults

\section{CONCLUSIONS}

In this paper, two identification approaches for robust fault detection are presented: namely the worst-case and set-membership approaches. The worst-case approach considers parametric uncertainty and additive noise. On the other hand, the setmembership approach considers only uncertainty due to additive noise but, by means of introducing an extra variable noise, can be considered variation in parameters. These different assumptions on uncertainty sources leads to different parameter uncertain sets. When applying these models to fault detection every identification approach should be used with the associated fault detection test: The model obtained using the worst-case approach with the direct test since it is based on propagating the parameter uncertainty to the residual or predicted output, while the model obtained using the set-membership approach is associated with the inverse test since it is based on checking if there is a value inside the model parameter uncertainty set that can explain the measured output. In the inverse test two approximations can be used: The outer approximation, conservative that can mask some faults, and the inner approximation, restrictive that can indicate a fault when no occurs. The two tests have different behaviours, defined by their sensitivities, in front of additive and multiplicative faults. This characteristic could be used in the future with fault isolation and estimation purposes. Moreover it has been showed that the direct test presents better fault sensitivity with respect to additive faults while the inverse test with respect to multiplicative ones. A case study based on a four tanks system has been used to exemplify the properties of the two identification and associated fault detection approaches.

\section{REFERENCES}


Adrot, O., D. Maquin, J. Ragot, "Bounding approach to the fault detection of uncertain dynamic systems", Safeprocess 2000, Budapest. Hungary.

B. Bamieh, L. Giarré. (2002) "Identification of linear parameter varying models", Int. J. Robust Nonlinear Control, vol.12, pp.841-853.

S.P. Bhattacharyya, H. Chapellat, L.H. Keel. (1995) Robust Control. The Parametric Approach, Prentice Hall.

J. Blesa, V. Puig, J. Saludes. (2009a) Identification for Passive Robust Fault Detection of LPV Systems using Zonotopes. In Proccedings of 7th IFAC Symposium on Fault Detection, Supervision and Safety of Technical Processes (SAFEPROCESS'09) Barcelona, Spain.

J. Blesa, V. Puig, Y. Bolea. (2009b) Robust fault detection using interval LPV parity Equations: Application to an Open-flow Canal. In proccedings European Control Conference (ECC’09) Budapest. Hungary.

J.M Bravo, T. Alamo, E.F. Camacho. (2006) "Bounded error identification of systems with time-varying parameters." IEEE Transactions on Automatic Control, Volume 51, Issue 7, 1144 - 1150.

G. Calafiore, M. C. Campi, L. El Ghaoui. (2002) Identification of reliable predictor models for unknown systems: A dataconsistency approach based on learning theory, In Proceedings of the 15th IFAC world congress.

M.C. Campi, G. Calafiore, S., Garatti. (2009) “Interval predictor models: Identification and reliability", Automatica, Volume 45, Issue 8. pp. 382-392.

C. Combastel, Q. Zhang and A. Lalami (2008). Fault diagnosis based on the enclosure of parameters estimated with an adaptive observer. In Proccedings of $17^{\text {th }}$ IFAC World Congress, Seoul, Korea.

C. Combastel, S.A. Raka (2009). A Set-Membership Fault Detection Test with Guaranteed Robustness to Parametric Uncertainties in Continuous Time Linear Dynamical Systems. In Proccedings of 7th IFAC Symposium on Fault Detection, Supervision and Safety of Technical Processes (SAFEPROCESS’09) Barcelona, Spain.

J. Chen, R.J. Patton. (1999) Robust Model-Based Fault Diagnosis for Dynamic Systems, Kluwer Academic Publishers.

I. Fagarasan, S. Ploix, S. Genti. (2004) Causal fault detection and isolation based on a set-membership approach. Automatica, 40 (12). 2099-2110.

P. Guerra, V. Puig. (2008) "Passive Robust Fault Detection using Interval MA Parity Equations: Inverse vs Direct Image Tests". Proceedings of the 17th World Congress IFAC. Seoul, Korea.

A. Ingimundarson, V. Puig, T. Álamo, J.M Bravo and P. Guerra. (2008) Robust fault detection using zonotope-based setmembership consistency test. Journal of Adaptive Control and Signal Processing, 23(4): 311-330.

R. Isermann (2006). Fault-diagnosis systems: An introduction from fault detection to fault tolerance, Berlin: Springer. 
K.H. Johansson. (2000) “The quadruple-tank process: A multivariable laboratory with an adjustable zero". IEEE Transactions on Control Systems Technology, 8(3):456-465.

M. Milanese, J. Norton, H. Piet-Lahanier, E. Walter (eds). (1996) “Bounding Approaches to System Identification”. Plenum Press.H.

L. Montgomery. (1989) “Computing the volume of a zonotope,” Amer. Math. Monthly, vol. 96, p. 431.

S. Ploix, O. Adrot, J. Ragot. (1999) "Parameter Uncertainty Computation in Static Linear Models", 38th IEEE Conference on Decision and Control, Phoenix, Arizona, USA, 1999.

S. Ploix, O. Adrot. (2006). Parity relations for linear uncertain dynamic systems. Automatica, 42 (9), 1553-1562.

V. Puig, J. Quevedo, T. Escobet, F. Nejjari, S. de las Heras. (2008) "Passive Robust Fault Detection of Dynamic Processes Using Interval Models”, IEEE Transactions on Control Systems Technology, Volume 16, Issue 5, 1083 - 1089.

V. Puig, A. Ingimundarson, and S. Tornil. Robust fault detection using inverse images of interval functions. In Proceedings of IFAC SAFEPROCESS 2006, Beijing, China, 2006.

W. Reinelt, A. Garulli, L. Ljung. (2002) “Comparing different approaches to model error modeling in robust identification”. Automatica, 38, pp. 787-803.

M. Á. Sainz, J. Armengol, J. Vehí. (2002). Fault detection and isolation of the three-tank system using the modal interval Analysis. Journal of Process Control, 12 (2), 325-338.

A. Vicino and G. Zappa (1996) "Sequential aproximation of feasible parameter sets for identification with set membership uncertainty," IEEE Trans. Autom. Control, vol. 41, no. 6, pp. 774-785.

\section{APPENDIX}

Proposition 1. The maximum and minimum prediction values provided by model (1) are given by

$$
\begin{aligned}
& \overline{\hat{y}}(k)=\hat{y}^{0}(k)+\|\varphi(k) \mathbf{H}\|_{1} \\
& \underline{\hat{y}}(k)=\hat{y}^{0}(k)-\|\varphi(k) \mathbf{H}\|_{1}
\end{aligned}
$$

considering that uncertain parameters $\boldsymbol{\theta} \in \boldsymbol{\Theta}$ where $\boldsymbol{\Theta}$ is a zonotope parameterised as in (2) and $\hat{y}^{0}(k)$ is the model output prediction with nominal parameters: $\hat{y}^{0}(k)=\boldsymbol{\varphi}(k) \boldsymbol{\theta}^{0} \quad$ where $\quad \boldsymbol{\theta}^{0}=\left(\theta_{1}^{0}, \ldots, \theta_{n_{\theta}}^{0}\right)$. 
Proof. Taking into account that uncertain parameters $\boldsymbol{\theta}$ belong to the zonotope $\boldsymbol{\Theta}$ parameterised as in (2), the prediction provided by the model (1) can be written as follows

$$
\hat{y}(k)=\boldsymbol{\varphi}(k)\left(\boldsymbol{\theta}^{0}+\mathbf{H z}\right)=\boldsymbol{\varphi}(k) \boldsymbol{\theta}^{0}+(\boldsymbol{\varphi}(k) \mathbf{H}) \mathbf{z}=\boldsymbol{\varphi}(k) \boldsymbol{\theta}^{0}+\sum_{i=1}^{n_{\theta}} \boldsymbol{\varphi}_{i}(k)\left(\sum_{j=1}^{n} \mathbf{H}_{i j} \mathbf{z}_{j}\right)=\boldsymbol{\varphi}(k) \boldsymbol{\theta}^{0}+\sum_{j=1}^{n} \mathbf{z}_{j}\left(\sum_{i=1}^{n_{\theta}} \boldsymbol{\varphi}_{i}(k) \mathbf{H}_{i j}\right)
$$

Then, $\overline{\hat{y}}(k)=\max _{\boldsymbol{\theta} \in \boldsymbol{\Theta}} \boldsymbol{\varphi}(k) \boldsymbol{\theta}$ is obtained for $\mathbf{z}_{j}=\operatorname{sign}\left(\sum_{i=1}^{n_{\theta}} \boldsymbol{\varphi}_{i}(k) \mathbf{H}_{i j}\right)$ and can be expressed as follows

$$
\overline{\hat{y}}(k)=\boldsymbol{\varphi}(k) \boldsymbol{\theta}^{0}+\sum_{j=1}^{n}\left|\sum_{i=1}^{n_{\theta}} \boldsymbol{\varphi}_{i}(k) \mathbf{H}_{i j}\right|=\hat{y}^{0}(k)+\|\boldsymbol{\varphi}(k) \mathbf{H}\|_{1}
$$

Analogously, $\underline{\hat{y}}(k)=\min _{\boldsymbol{\theta} \in \boldsymbol{\Theta}} \boldsymbol{\varphi}(k) \boldsymbol{\theta}$ is obtained when $\mathbf{z}_{j}=-\operatorname{sign}\left(\sum_{i=1}^{n_{\theta}} \boldsymbol{\varphi}_{i}(k) \mathbf{H}_{i j}\right)$ leading to the following expression

$$
\underline{\hat{y}}(k)=\boldsymbol{\varphi}(k) \boldsymbol{\theta}^{0}-\sum_{j=1}^{n}\left|\sum_{i=1}^{n_{\theta}} \boldsymbol{\varphi}_{i}(k) \mathbf{H}_{i j}\right|=\hat{y}^{0}(k)-\|\boldsymbol{\varphi}(k) \mathbf{H}\|_{1}
$$

This completes the proof.

Proposition 2. Given a sequence of $M$ output measurements $y(k)$ and regressor values $\varphi(k)$, considering model (1) with parameters $\boldsymbol{\theta}$ belonging to the set $\boldsymbol{\Theta}$, the comparison of worst-case and set-membership conditions given by (4) and (7) considering different bounds of additive noise $\sigma_{w c}$ and $\sigma_{s m}$, respectively. Leads to

$$
\sigma_{s m}=2\|\varphi(k) \mathbf{H}\|_{1}+\sigma_{w c}
$$

Proof. The set-membership conditions (7) can be rewritten $\forall k=1, \ldots, M$ as follows

$$
y(k) \leq \underline{\hat{y}}(k)+\sigma_{s m} \text { and } \quad y(k) \geq \overline{\hat{y}}(k)-\sigma_{s m}
$$

Analogously, worst-case conditions (4) can be rewritten $\forall k=1, \ldots, M$ as follows

$$
y(k) \leq \overline{\hat{y}}(k)+\sigma_{w c} \text { and } y(k) \geq \underline{\hat{y}}(k)-\sigma_{w c}
$$


Both conditions (55) and (56) are equivalent when

$$
\overline{\hat{y}}(k)+\sigma_{w c}=\underline{\hat{y}}(k)+\sigma_{s m} \text { and } \underline{\hat{y}}(k)-\sigma_{w c}=\overline{\hat{y}}(k)-\sigma_{s m}
$$

that leads to the single condition

$$
\sigma_{s m}=\overline{\hat{y}}(k)-\underline{\hat{y}}(k)+\sigma_{w c}
$$

Finally, (54) follows from (58) taking into account that $\overline{\hat{y}}(k)-\underline{\hat{y}}(k)=2\|\boldsymbol{\varphi}(k) \mathbf{H}\|_{1}$ from Proposition 1. This completes the proof.

Outer approximation of the Feasible Parameter Set (FPS) using zonotopes (Bravo et al. 2006)

$\mathbf{F}_{k}=\left\{\boldsymbol{\theta} \in \mathbb{R}^{n_{\theta}} \mid y(k)-\sigma \leq \boldsymbol{\varphi}(k) \boldsymbol{\theta} \leq y(k)+\sigma\right\}$

$\mathbf{A F P S}_{k}=\boldsymbol{\theta}_{k}^{0} \oplus \boldsymbol{X}_{k} \mathbf{B}^{n}$ with $\boldsymbol{X}_{k}=\left[\mathbf{x}_{1}^{k} \mathbf{x}_{2}^{k} \ldots \mathbf{x}_{n}^{k}\right]$

$\mathbf{v}(j) \oplus \mathbf{T}(j) \mathbf{B}^{n} \supseteq \mathbf{A F P S}_{k} \cap \mathbf{F}_{k}$

Where

$\mathbf{v}(j)=\left\{\begin{array}{cc}\boldsymbol{\theta}_{k}^{0}+\left(\frac{y(k)-\varphi(k) \boldsymbol{\theta}_{k}^{0}}{\varphi(k) \mathbf{x}_{j}^{k}}\right), & \text { if } 1 \leq j \leq n \text { and } \varphi(k) \mathbf{x}_{j}^{k} \neq 0 \\ \boldsymbol{\theta}_{k}^{0}, & \text { otherwise }\end{array}\right.$

$\mathbf{T}(j)=\left\{\begin{array}{cc}{\left[\mathbf{t}_{t}^{j} \mathbf{t}_{2}^{j} \ldots \mathbf{t}_{n}^{j}\right],} & \text { if } 1 \leq j \leq n \text { and } \varphi(k) \mathbf{x}_{j}^{k} \neq 0 \\ \mathbf{X}_{k}, & \text { otherwise }\end{array}\right.$

$\mathbf{T}_{i}^{j}=\left\{\begin{array}{cc}\mathbf{x}_{i}^{k}-\left(\frac{\varphi(k) \mathbf{x}_{i}^{k}}{\varphi(k) \mathbf{x}_{j}^{k}}\right) \mathbf{x}_{j}^{k}, & \text { if } i \neq j \\ \left(\frac{\sigma}{\varphi(k) \mathbf{x}_{j}^{k}}\right) \mathbf{x}_{j}^{k}, & \text { if } i=j\end{array}\right.$

Then $\mathbf{A F P S}_{k+1} \supseteq \mathbf{A F P S}_{k} \cap \mathbf{F}_{k}$

with $\mathbf{A F P S}_{k+1}=\boldsymbol{\theta}_{k+1}^{0} \oplus \boldsymbol{X}_{k+1} \mathbf{B}^{n}$,

where $\boldsymbol{\theta}_{k+1}^{0}=\mathbf{v}\left(j^{*}\right), \quad \boldsymbol{X}_{k+1}=\mathbf{T}\left(j^{*}\right), j^{*}=\arg \min _{0 \leq j \leq n} \operatorname{vol}\left(\mathbf{v}(j) \oplus \mathbf{T}(j) \mathbf{B}^{n}\right)$ 